\title{
Metal Matrix Syntactic Foams: Manufacture, Matrix Material, Microstructure, Modulus and More
}

\author{
Yuyuan Zhao
}

Syntactic foams are particulate composite materials that have hollow or porous ceramic particles embedded in continuous polymer or metal matrices. They can have very different behaviors under compression or impact loading conditions, depending on whether the porosity enclosed in the ceramic particles plays a significant role or not. If the ceramic particles are intact during loading, syntactic foams are essentially the same as conventional particulate-reinforced composites, apart from lower densities, and are often called composites instead of syntactic foams. If the ceramic particles in the syntactic foams collapse or deform during loading, however, the theoretical and experimental analyses used for characterizing the mechanical behavior of particulate composites can no longer be applied. In the latter case, syntactic foams behave more like solid foams (porous solids) with some unique characteristics. They deserve to be treated as a distinctive class of materials.

Metal matrix syntactic foams have not been studied as extensively as polymer matrix syntactic foams, which have been commercially available for several decades. This is largely because they are more difficult and costly to manufacture and the benefits accrued from incorporating hollow or porous particles are less pronounced than polymer matrix syntactic foams, especially in terms of mechanical properties.

Metal matrix syntactic foams have potential for many applications. The majority of the metal matrix syntactic foams developed so far are intended for structural applications where either weight reduction or impact energy absorption is desired.

Several combinations of matrix materials and ceramic particles have been studied previously. Aluminum and magnesium alloy matrices are generally used, and syntactic foams with steel or titanium matrices have also been developed. There are very limited choices of hollow or porous ceramic particles, including microballoons, cenospheres, and E-spheres, which are all silicaand alumina-based. Very little, if any, research has been conducted on other systems. The availability of high-quality ceramic particles will be crucial for the development of new metal matrix syntactic foams.

The properties of metal matrix syntactic foams depend not only on the matrix materials but on the type, composition, volume percentage, and distribution of the ceramic particles. All these factors dictate the choice of cost-effective manufacturing technologies, which have a significant influence on the quality and properties of the resultant materials. Research so far has focused on developing manufacturing technologies for combinations of matrix materials and ceramic particles, as well as on the characterizing the properties of the asmanufactured syntactic foams.

No overviews on the research in metal matrix syntactic foams exist in the literature. The timely article by Pradeep $\mathrm{K}$. Rohatgi et al. fills this gap. It reviews the current developments in metal matrix syntactic foams, encompassing the common types of ceramic particles, manufacturing methods, microstructure, compressive properties, and applications. Because of space constraints, some research topics (e.g., load partitioning and failure mechanisms) are not covered. Nevertheless, this article serves as an excellent starting point to familiarize oneself with metal matrix syntactic foams.

The paper by Xiaobing Xue and Yuyuan Zhao studies the integrity and elastic modulus of a newly developed material, titanium matrix syntactic foam. Unlike aluminum or magnesium matrix syntactic foams, which can be manufactured by melt infiltration or casting, syntactic foams with highmelting-point or reactive matrices often necessitate a solid state manufacturing route. In the case of titanium, powder metallurgy is an obvious choice. A major problem of using powder metallurgy for manufacturing syntactic foams is that the hollow or porous ceramic particles may be damaged during compaction. To address this issue, the paper describes quantitative methods to assess the proportion of the damaged particles.

The last two papers by Dung D. Luong et al. study the high strain rate compression of magnesium and aluminum alloy matrix composites reinforced with hollow cenospheres. It is shown that syntactic foams have better energy absorption capabilities than the matrix materials and high strain rates result in higher energy absorption.

Yuyuan Zhao is with the School of Engineering at the University of Liverpool, U.K. and is the advisor to JOM from the Composite Materials Committee of the Structural Materials Division of TMS.

\section{Yuyuan Zhao is a TMS Member!}

To read more about him, turn to page 14. To join TMS, visit www.tms.org/Society/Membership.aspx. 